Case Report

\title{
Bilateral Simultaneous Anterior Ischemic Optic Neuropathy following Total Knee Arthroplasty with Epidural Block
}

\author{
Ahmed M. Habib $\mathbb{D}^{1,2}$ Yousef A. Fouad $\mathbb{D}^{1,2}$ and Mahmood O. Mekkawy ${ }^{1}$ \\ ${ }^{1}$ Al Mashreq Eye Center, Cairo, Egypt \\ ${ }^{2}$ Department of Ophthalmology, Ain Shams University Hospitals, Cairo, Egypt
}

Correspondence should be addressed to Yousef A. Fouad; yousef.a.fouad@gmail.com

Received 5 March 2021; Accepted 29 May 2021; Published 8 June 2021

Academic Editor: Takaaki Hayashi

Copyright (c) 2021 Ahmed M. Habib et al. This is an open access article distributed under the Creative Commons Attribution License, which permits unrestricted use, distribution, and reproduction in any medium, provided the original work is properly cited.

\begin{abstract}
Ischemic optic neuropathy (ION) resulting in perioperative vision loss (POVL) is a rare occurrence following nonocular procedures. Bilateral simultaneous anterior ION (AION) is even rarer, and no cases have been reported after central neuraxial block. We report a case of bilateral simultaneous AION, confirmed by multimodal imaging, in a 66-year-old male patient who underwent total knee arthroplasty under epidural anesthesia in which episodes of hypotension-one intraoperatively and one late postoperatively-had occurred. Hypotension is the most common adverse effect to epidural block, and counseling about POVL should extend beyond general anesthesia to include those undergoing procedures with central neuraxial block.
\end{abstract}

\section{Introduction}

Perioperative vision loss (POVL) is a rare but catastrophic complication of nonocular surgical procedures, for which ischemic optic neuropathy (ION)-particularly posterior ION (PION)-is most commonly implicated [1]. The frequency of permanent POVL complicating noncardiac surgery was estimated to be 1 in 118,783 patients in a large retrospective analysis, with all affected cases having undergone general anesthesia and with all ION cases having developed PION [2]. More scarce are reports of POVL with the use of central neuraxial block [3] and POVL due to anterior ION (AION) [4].

Reported risk factors for POVL due to ION are related to perioperative hemodynamic instability, including prolonged surgical duration, anemia, hypotension, and blood transfusion [1]. The most commonly implicated surgeries are cardiothoracic and orthopedic ones, especially spine procedures [2]. The ensuing PION or AION may be unilateral or bilateral [4], and permanent severe visual loss is the final fate in at least $75 \%$ of the cases [1].

\section{Case Report}

A 66-year-old male patient underwent total knee arthroplasty under epidural anesthesia for severe osteoarthritis. The patient had a medical history of controlled hypertension and Type II diabetes mellitus and a stroke in the preceding year that focally involved the right frontal lobe, with minimal residual neurological impairment for which the patient had received the proper urgent management and was maintained on daily clopidogrel $(75 \mathrm{mg})$ and aspirin $(75 \mathrm{mg})$. Ophthalmological examination one month prior to the surgery had shown a corrected distance visual acuity (CDVA) of 20/20 (1.0) in both eyes with a hyperopic refraction of $+1.00 \mathrm{D}$ in the right eye and $+1.50 /-0.75 \times 130$ in the left eye. No abnormality had been detected on fundus examination except for mild nonproliferative diabetic retinopathy.

The surgery was uneventful with minimal blood loss and a duration of around 90 minutes. There was a brief episode of hypotension $(80 / 50 \mathrm{mmHg})$ following the introduction of the epidural anesthesia, but the blood pressure returned to normal levels with the aid of vasopressors. The immediate 


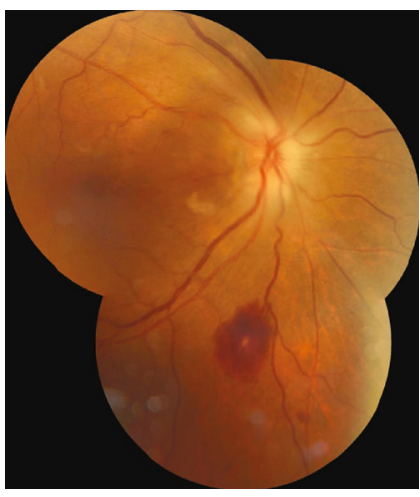

(a)

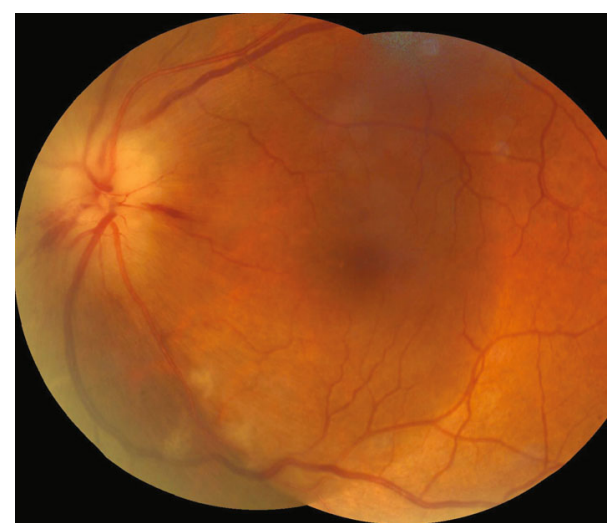

(b)

Figure 1: Fundus photography of the right (a) and left (b) eyes showing pale, edematous optic discs, cotton wool patches, and intraretinal hemorrhages.

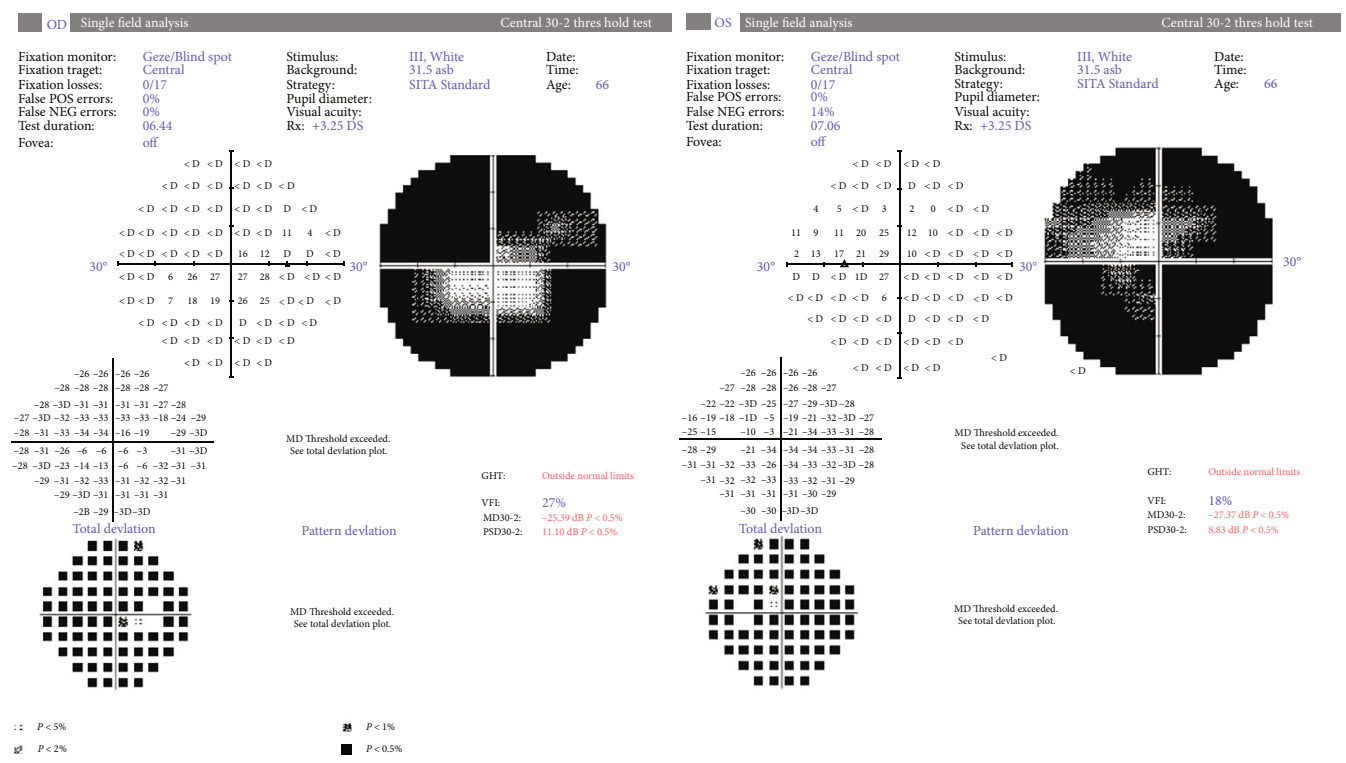

(a)
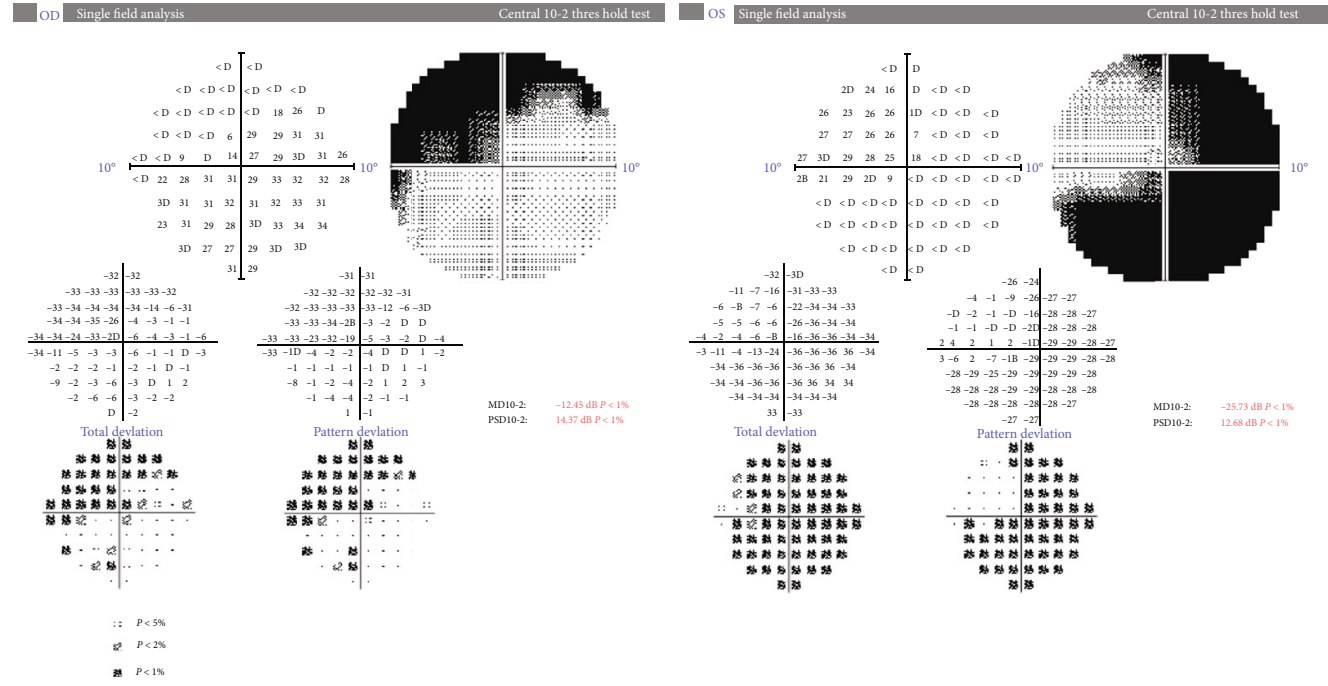

(b)

FIGURE 2: 30-2 (a) and central 10-2 (b) visual field plots of both eyes of the patient showing tubular fields with small, central remnant islands of vision. 

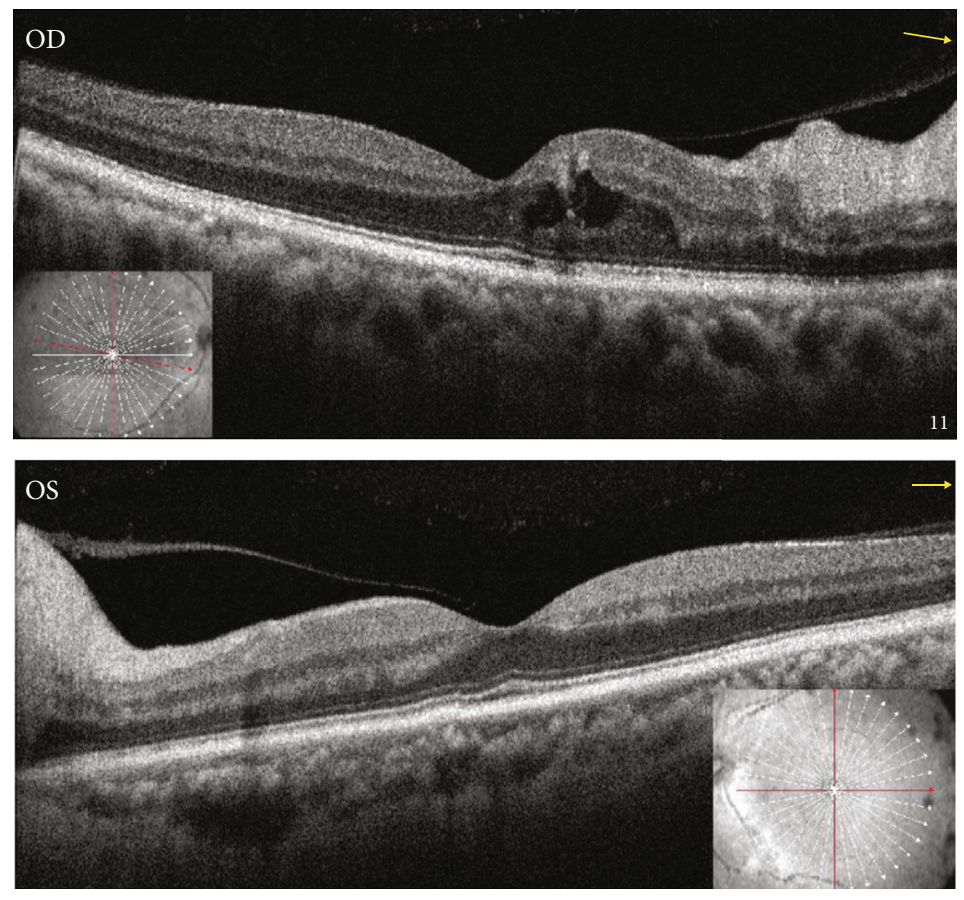

FIGURE 3: OCT of the right (a) and left (b) maculae showing preserved foveal architecture but distorted and thickened peripapillary nerve fiber layer, more pronounced in the right eye.
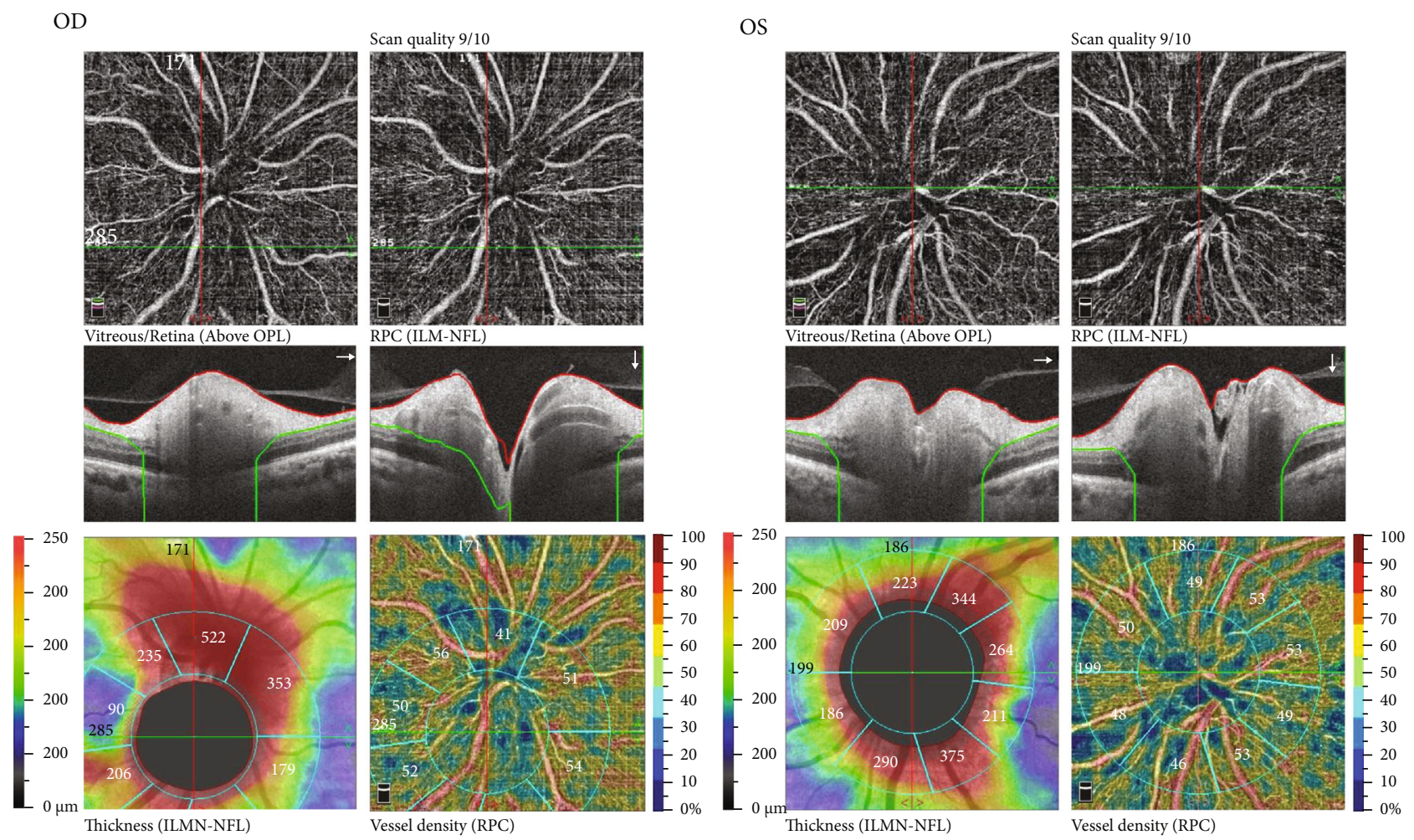

FIGURE 4: OCT-A of the right and left eyes showing peripapillary distortion of the retinal nerve fiber layer and pronounced loss of the capillary microvascular perfusion.

postoperative period was uneventful. On the first postoperative night, the patient experienced another attack of hypotension with the blood pressure dropping down to $70 / 40 \mathrm{mmHg}$; this was successfully managed with the administration of vasopressors and fluids. On the first morning after the surgery, the patient experienced blurring of vision with large blank areas in his field of vision. Magnetic resonance imaging was ordered but revealed no acute cerebral insults. 
On ophthalmological examination, the right and left eyes had a CDVA of 20/80 (0.6) and 20/20 (1.0), and an intraocular pressure of $16 \mathrm{mmHg}$ and $21 \mathrm{mmHg}$, respectively. Both pupils were sluggish to react to light stimuli, but the anterior segments were otherwise free. Fundus examination (Figure 1) revealed bilateral swollen, pale optic discs, with peripapillary cotton wool spots, intraretinal hemorrhages in the right fundus (Figure 1(a)), and retinal nerve fiber layer hemorrhages in the left fundus (splinter hemorrhages, Figure 1(b)). Visual field examination was ordered (Figure 2) and revealed severe constriction in the fields of both eyes (tubular fields) on the 30-2 exams (Figure 2(a)). Central 10 degrees examination (Figure 2(b)) confirmed the presence of small remnant islands in the central fields of both eyes. Optical coherence tomography (OCT) of the macula (Figure 3) revealed preserved foveal anatomy, with thickening and distortion of the peripapillary retinal nerve fiver layer which, in the right eye, was more pronounced and associated with intraretinal edema. OCTAngiography (OCT-A) of the optic disc (Figure 4) showed diffuse distortion and loss of the peripapillary microvascular cuff in both eyes. No symptoms of arteritis (headache or jaw claudication) were elicited on detailed history taking from the patient, and an ordered erythrocytic sedimentation was within normal values. This led to a diagnosis of bilateral nonarteritic AION. A follow-up of the patient after 1 month did not reveal any change except for mild subjective visual improvement reported by the patient.

\section{Discussion}

To the best of our knowledge, this is the first report on bilateral simultaneous ION following surgery with central neuraxial block. Only one other report [3] exists in the literature that describes unilateral ION that occurred after epidural block for emergency cesarean section, in which a hypotensive episode had occurred on administration of the anesthetic. Bilateral simultaneous AION has, however, been reported to occur spontaneously [5], after ingestion of sildenafil [6, 7], with hypercoagulability [8] and vasculitis [9], with longterm hemodialysis [10], and after prostatic resection surgery under general anesthesia [11].

In our described case, both intraoperative and delayed postoperative hypotension could be implicated in the development of ION. Perioperative hypotension is recognized as a potential risk for developing POVL due to $\mathrm{ION}^{12}$ and also as the most common complication of epidural block [12]. Physiological drop in blood pressure often occurs within the initial 30 minutes after introduction of the anesthetic and is thought to majorly be attributed to the sympathetic blockade resulting in arteriolar and venular dilation and an ensuing "functional hypovolemia." This has been reported to respond excellently to vasopressors [12]. On the other hand, the use of vasopressors has been suggested as a-statistical-risk factor for developing POVL [13]. The debate around how significant of a role hemodynamic changes play in POVL is still ongoing [14].

Other risk factors for developing POVL include prolonged surgical procedure, significant blood loss or the need for blood transfusion, anemia, facial edema, pressure on the eyes, and/or prone head positioning intraoperatively [1], none of which had occurred with the case we report. The lack of prospective study design and the reliance on retrospective analysis of large datasets reduce the strength of evidence on risk factors for developing POVL. Nevertheless, the American Society of Anesthesiologists Task Force on Perioperative Visual Loss advises on (1) the continuous intraoperative monitoring of blood pressure with conservation in usage of deliberate hypotensive techniques and vasopressors on a case-by-case basis, (2) use of colloids and crystalloids for patients with substantial blood loss, with continuous monitoring of central venous pressure and hemoglobin/hematocrit values, (3) avoiding eye pressure and maintaining elevated head position whenever possible, and (4) immediate postoperative check on patient's vision after regaining of an alert state and immediate ophthalmological consultation on early suspicion of POVL [15].

Counseling of patients about the minimal risk of developing POVL, especially those with identifiable preoperative medical risk factors, has been recommended for patients scheduled for general anesthesia [1]. Based on the aforementioned case, we conclude that counseling should expand to include those undergoing procedures with central neuraxial block.

\section{Data Availability}

No data were used to support this study.

\section{Conflicts of Interest}

The authors declare that they have no conflicts of interest.

\section{References}

[1] B. Chwalisz, A. L. Gilbert, and J. W. Gittinger, "Perioperative vision loss after non-ocular surgery," Seminars in Ophthalmology, vol. 33, no. 1, pp. 17-22, 2018.

[2] M. E. Warner, M. A. Warner, J. A. Garrity, R. A. MacKenzie, and D. O. Warner, "The frequency of perioperative vision loss," Anesthesia and Analgesia, vol. 93, no. 6, pp. 14171421, 2001.

[3] M. Gupta, P. Puri, and I. G. Rennie, "Anterior ischemic optic neuropathy after emergency caesarean section under epidural anesthesia," Acta Anaesthesiologica Scandinavica, vol. 46, no. 6, pp. 751-752, 2002.

[4] A. Goyal, M. Elminawy, M. A. Alvi et al., "Ischemic optic neuropathy following spine surgery: case control analysis and systematic review of the literature," Spine (Phila Pa 1976), vol. 44, no. 15, pp. 1087-1096, 2019.

[5] M. J. Goff, "Bilateral simultaneous anterior ischemic optic neuropathy in a young, healthy man," Archives of Ophthalmology, vol. 121, no. 11, pp. 1652-1653, 2003.

[6] M. M. Moschos and I. Margetis, "Bilateral simultaneous anterior ischemic optic neuropathy associated with sildenafil," Case Reports in Ophthalmology, vol. 2, no. 2, pp. 262-265, 2011.

[7] A. Tarantini, A. Faraoni, F. Menchini, and P. Lanzetta, "Bilateral simultaneous nonarteritic anterior ischemic optic neuropathy after ingestion of sildenafil for erectile dysfunction," Case 
Reports in Medicine, vol. 2012, Article ID 747658, 5 pages, 2012.

[8] M. S. Tönz, V. Rigamonti, and M. E. Iliev, "Simultaneous, bilateral anterior ischemic optic neuropathy (AION) in polycythemia vera: a case report," Klinische Monatsblätter für Augenheilkunde, vol. 225, no. 5, pp. 504-506, 2008.

[9] M. Hata, K. Miyamoto, A. Oishi, and N. Yoshimura, "Case of simultaneous bilateral anterior ischemic optic neuropathy associated with Takayasu disease," Canadian Journal of Ophthalmology, vol. 48, no. 2, pp. e33-e35, 2013.

[10] U. Limon, B. I. S. Akcay, and E. Akcay, "Bilateral simultaneous non-arteritic anterior ischemic optic neuropathy with occlusion of unilateral cilioretinal artery: a case report," Journal of Northern Clinics of Istanbul, vol. 6, no. 4, pp. 407-411, 2019.

[11] L. M. Sadaba, A. Garcia-Layana, M. J. Maldonado, and J. M. Berian, "Bilateral ischemic optic neuropathy after transurethral prostatic resection: a case report," BMC Ophthalmology, vol. 6, no. 1, p. 32, 2006.

[12] K. Holte, N. B. Foss, C. Svensén, C. Lund, J. L. Madsen, and H. Kehlet, "Epidural anesthesia, hypotension, and changes in intravascular volume," Anesthesiology, vol. 100, no. 2, pp. 281-286, 2004.

[13] M. A. Myers, S. R. Hamilton, A. J. Bogosian, C. H. Smith, and T. A. Wagner, "Visual loss as a complication of spine surgery," Spine (Phila Pa 1976), vol. 22, no. 12, pp. 1325-1329, 1997.

[14] R. Foroozan and K. C. Golnik, "Are anemia and hypotension causally related to perioperative ischemic optic neuropathy?," Journal of Neuro-Ophthalmology, vol. 37, no. 1, pp. 81-86, 2017.

[15] American Society of Anesthesiologists Task Force on Perioperative Visual Loss, "Practice advisory for perioperative visual loss associated with spine surgery: an updated report by the American Society of Anesthesiologists Task Force on Perioperative Visual Loss," Anesthesiology, vol. 116, no. 2, pp. 274$285,2012$. 